\title{
Oral Dexmedetomidine Versus Midazolam as Anesthetic Premedication in Children Undergoing Congenital Heart Surgery
}

\author{
Seyedeh Zahra Faritus ${ }^{1}$; Mehrdad Khazaee-Koohpar ${ }^{1}$; Mohsen Ziyaeifard ${ }^{1,{ }^{*}}$; Mohammad \\ Javad Mehrabanian ${ }^{1}$ \\ ${ }^{1}$ Rajaie Cardiovascular, Medical and Research Center, Iran University of Medical Sciences, Tehran, Iran \\ *Corresponding author: Mohsen Ziyaeifard, Rajaie Cardiovascular, Medical and Research Center, Iran University of Medical Sciences, Tehran, Iran. Tel: +98-2122043921, Fax: +98-2122042026, \\ E-mail: mziyaeifard@yahoo.com
}

Received: October 31, 2014; Revised: December 7, 2014; Accepted: December 24, 2014

\begin{abstract}
Background: Premedication is required for reducing anxiety and child's struggling against mask acceptance on anesthesia in pediatric surgery for congenital heart disease. Midazolam has been widely used for this purpose, but because of its side effects, finding an effective replacement with less complication is necessary.

Objectives: In the present study, we compared the efficacy of oral midazolam versus dexmedetomidine in terms of anxiolysis and mask acceptance behavior.

Patients and Methods: Sixty children aged between 2 and 12 years, and scheduled for on-pump surgery due to a congenital heart disease were randomly assigned into two groups. Oral midazolam ( $0.5 \mathrm{mg} / \mathrm{kg}$ up to $15 \mathrm{mg}$ per patient) and dexmedetomidine $(2 \mu \mathrm{gg} / \mathrm{kg}) \mathrm{were}$ administered 45 minutes pre-anesthesia. Children's anxiety, mask acceptance behavior, hemodynamic measures, and cardiopulmonary outcomes were recorded and compared.

Results: The mean age of patients was $3.96 \pm 2.04$ years. Twenty-eight (46.7\%) patients were females. Two drugs have similar effects on cardiopulmonary outcomes and hemodynamic measures $(\mathrm{P}>0.05)$. They equally relieved the children's anxiety (mean sedation score 1.93 \pm 0.63 and $2.0 \pm 0.63$ for midazolam and dexmedetomidine groups, respectively; $P>0.05$ ), while dexmedetomidine showed a better effect on improving the mask acceptance behavior (mean mask acceptance score $2.58 \pm 0.6$ and $1.6 \pm 0.67$ for midazolam and dexmedetomidine, respectively; $\mathrm{P}<0.05)$.

Conclusions: It appears reasonable to apply oral premedication with dexmedetomidine 45 minutes before transferring the patient to the operating room when he or she is more prone to resist inhalation anesthesia induction.
\end{abstract}

Keywords: Anesthesia; Inhalation; Midazolam; Analgesics; Anti-Anxiety Agents; Premedication; Dexmedetomidine

\section{Background}

Congenital heart defects are amongst the major health problems, which pose a great deal of pain and costs on patients, their family, and health system $(1,2)$. They are the most common birth defects and the leading cause of mortality amidst birth defect-related deaths (1). Surgical operation to correct the malformations is implicated in symptomatic cases where drug therapy fails. Surgical operation is performed under general anesthesia (3). Thus, anesthesia practice is a major contributor to the outcomes of surgical operation. Distress and the psychological trauma as maternal deprivation sequelae are major challenges in the practice of pediatric anesthesia (3). Oral midazolam, the most common anesthetic premedication in children, is being widely used to ease the children's separation anxiety during maternal deprivation on transfer into the operation room (4).

Despite several advantages of midazolam for anesthetic premedication in children, some of its side effects such as paradoxical reaction, restlessness, and other unfavorable behavioral changes (post-surgery) have limited its application as an ideal option (5). Therefore, suitable substitutes are sought with less unfavorable side effects. Dexmedetomidine, an $\alpha 2$-agonist with better pharmacokinetic properties and higher selectivity for $\alpha 2$-adrenoceptor than midazolam is under clinical investigation in various settings as a replacement for midazolam (4-8). It has been demonstrated in controlled trials that preoperational administration of dexmedetomidine favors midazolam premedication in terms of effective sedation induction upon parent separation and mask acceptance (9). However, the safety and efficacy of oral dexmedetomidine as premedication in congenital heart disease patients undergoing surgical operation has been poorly studied.

\section{Objectives}

In the present study, we tried to compare the effects of oral midazolam, including sedative effects and anxiolytic effects, with oral dexmedetomidine in pediatric patients who underwent surgical operation for congenital heart disease.

Copyright (C) 2015, Iranian Society of Regional Anesthesia and Pain Medicine(ISRAPM). This is an open-access article distributed under the terms of the Creative Commons Attribution-NonCommercial 4.0 International License (http://creativecommons.org/licenses/by-nc/4.0/) which permits copy and redistribute the material just in noncommercial usages, provided the original work is properly cited. 


\section{Patients and Methods}

\subsection{Study Design}

In this double-blinded randomized controlled trial, pediatric patients, aged 2 to 12 years, suffering from congenital heart disease and indicated for open heart on-pump surgical operation were recruited. Patients with hepatic or renal complications, conduction disorders, coronary artery disease, emergency operations and other complications or mental disabilities were excluded. Block randomization was used for allocation of patients into two groups; one group received midazolam and the other received dexmedetomidine.

Study performed in compliance with the Declaration of Helsinki as a statement of ethical principles for medical research involving human subjects. The research was approved by Institutional Review Board of Ethics at Rajaei Heart Center (RHC) (Tehran-Iran). The informed consent form was signed by the parents of all patients before entering the study.

\subsection{Drugs and Treatment Protocol}

Midazolam (Chemidarou, Iran) and dexmedetomidine (Precedex, Hospira, United States) were used to treat patients in the following treatment regimen. Oral midazolam ( $0.5 \mathrm{mg} / \mathrm{kg}$ up to $15 \mathrm{mg}$ per patient) and dexmedetomidine $(2 \mu \mathrm{g} / \mathrm{kg}$ ) were administered 45 minutes before anesthesia induction. The volume of the drugs was adjusted to $5 \mathrm{~mL}$ by adding 5\% dextrose solution. The patient's anxiety during separation from parents was scaled from 1 to 6 according to the Ramsay Sedation Scale(Table1) (10).A 4-point scoring system was used to evaluate the child's behavior at anesthesia induction and mask acceptance 45 minutes after premedication (Table 2). Anesthesia induction was performed using volatile gas, sevoflurane. Heart rate, respiratory rate and peripheral capillary oxygen saturation of each patient were measured and recorded carefully. The first measurement was performed before premedication and the obtained measure was considered as baseline. Second measurement was performed 20 minutes after medication and the third measurement at the time of entering the operation room. Other outcomes like nausea, vomiting, illusion, and other adverse outcomes were recorded carefully. All patients underwent the same protocol for anesthesia induction.

\begin{tabular}{|c|c|}
\hline Score & Description \\
\hline $\mathbf{1}$ & Anxious and agitated or restless, or both \\
\hline 2 & Cooperative, oriented, and calm \\
\hline 3 & Responsive to commands only \\
\hline 4 & $\begin{array}{l}\text { Exhibiting brisk response to light glabellar tap or loud audi- } \\
\text { tory stimulus }\end{array}$ \\
\hline 5 & $\begin{array}{l}\text { Exhibiting a sluggish response to light glabellar tap or loud } \\
\text { auditory stimulus }\end{array}$ \\
\hline 6 & Unresponsive \\
\hline
\end{tabular}

\begin{tabular}{ll}
\hline Table 2. & Mask Acceptance Behavior During Anesthesia Induction \\
\hline Score & Description \\
\hline $\mathbf{1}$ & Calm and cooperating \\
$\mathbf{2}$ & Anxious but without resistance \\
$\mathbf{3}$ & Anxious with slight resistance \\
$\mathbf{4}$ & Crying and/or struggling against mask \\
\hline
\end{tabular}

\subsection{Statistical Analysis}

All numerical data were represented as mean \pm standard deviation(SD) and the qualitative data as frequencies. Paired $t$ test was used to analyze the differences in each treatment group before and after treatment. Unpaired t test was used to compare the means between two treatment groups. For variables with non-normal distribution, Mann-Whitney U test was used for comparing the results between groups. To compare qualitative data, chi-square test was performed. P values less than 0.05 were considered as statistically significant. All statistical analysis was performed using SPSS software version 17 (IBM Corporation, United States).

\section{Results}

Our study included 30 patients receiving dexmedetomidine and 30 controls receiving midazolam as standard premedication for children anesthesia practice in Rajaei Heart Center (Tehran-Iran). The sample included 28 (46.7\%) females. The mean age of patients was $3.96 \pm 2.04$ years. Demographic information of the patients is presented in Table 3. Statistical analysis demonstrated no significant differences between groups with regard to demographic specifications, systolic and diastolic blood pressure, respiratory rate, and peripheral capillary oxygen saturation.

Analysis of the sedation scales, demonstrated that more percentage of patients in midazolam group are cooperative, oriented, and calm while more percentage of patients in dexmedetomidine group were responsive only to commands. However, these differences were not statistically significant $(P<0.05$; Figure 1$)$ and they equally relieved the children's anxiety (mean sedation score $1.93 \pm$ 0.63 and $2.0 \pm 0.63$ for midazolam and dexmedetomidine groups, respectively; $\mathrm{P}>0.05$ ).

Analysis of the mask acceptance behavior at anesthesia induction time revealed that more children receiving dexmedetomidine are calm and cooperate well in terms of mask acceptance while the number of patients with grade 2 of mask acceptance behavior, anxious but without resistance, were higher in midazolam group. These differences were statistically significant $(\mathrm{P}<0.05)$. Also, more patients in the midazolam group were categorized as grade 3 in mask acceptance behavior, and anxious with slight resistance. In sum, dexmedetomidine performed better in improving the mask acceptance behavior (mean mask acceptance score $2.58 \pm 0.6$ and $1.6 \pm 0.67$ for midazolam and dexmedetomidine, respectively; $\mathrm{P}<0.05$ ) (Figure 2 ).

In screening for adverse effect, we did not observe any case of hallucination, nausea, and vomiting in patients. One case of hiccups was observed in a patient receiving midazolam. 
Table 3. Demographic Information, Hemodynamic and Vital Sign Monitoring Testing Results of the Patients in Two Groups Receiving Midazolam (MIDA) and Dexmedetomidine (DEX) as Anxiolytic Premedication Before Anesthesia a, b

\begin{tabular}{|c|c|c|c|}
\hline Group & MIDA & DEX & PValue \\
\hline Age, y & $3.63 \pm 1.81$ & $4.28 \pm 2.22$ & 0.91 \\
\hline Weight, Kg & $13.80 \pm 4.49$ & $14.33 \pm 4.68$ & 0.28 \\
\hline Gender, female, \% & 36.7 & 63.3 & 0.195 \\
\hline SBP, before premedication, $\mathrm{mmHg}$ & $101.33 \pm 8.89$ & $99.17 \pm 7.507$ & 0.91 \\
\hline SBP, 20 minutes after premedication, $\mathrm{mmHg}$ & $96.33 \pm 10.16$ & $92.83 \pm 7.84$ & 0.14 \\
\hline SBP, 45 minutes after premedication, $\mathrm{mmHg}$ & $95.33 \pm 9.9$ & $92.5 \pm 7.96$ & 0.22 \\
\hline DBP, before premedication, mmHg & $58.83 \pm 9.53$ & $57.17 \pm 9.97$ & 0.93 \\
\hline DBP, 20 minutes after premedication, $\mathrm{mmHg}$ & $55.33 \pm 8.996$ & $52 \pm 9.43$ & 0.2 \\
\hline DBP, 45 minutes after premedication, $\mathbf{m m H g}$ & $53.67 \pm 8.6$ & $52.67 \pm 8.38$ & 0.59 \\
\hline HR, before premedication, beat/min & $111.43 \pm 11.58$ & $110.17 \pm 15.634$ & 0.72 \\
\hline HR, 20 minutes after premedication, beat/min & $108.03 \pm 9.586$ & $109.27 \pm 14.77$ & 0.70 \\
\hline HR, 45 minutes after premedication, beat/min & $108.37 \pm 10.46$ & $109.83 \pm 14.7$ & 0.65 \\
\hline $\mathrm{RR}$, before premedication, breath/min & $19 \pm 2.44$ & $18.47 \pm 2.446$ & 0.4 \\
\hline RR, 20 minutes after premedication, breath/min & $17.20 \pm 2.44$ & $17.07 \pm 2.01$ & 0.98 \\
\hline RR, 45 minutes after premedication, breath/min & $17.0 \pm 2.27$ & $17.0 \pm 1.94$ & 0.9 \\
\hline Sp02, before premedication, $\%$ & $89.93 \pm 6.64$ & $86.70 \pm 10.8$ & 0.16 \\
\hline Sp02, 20 minutes after premedication, $\%$ & $89.43 \pm 6.564$ & $87.37 \pm 10.42$ & 0.36 \\
\hline Sp02, 45 minutes after premedication, $\%$ & $89.23 \pm 6.479$ & $87.83 \pm 10.43$ & 0.92 \\
\hline
\end{tabular}

a abbreviation: DBP, diastolic blood pressure; HR, heart rate; RR, respiratory rate; SBP, systolic blood pressure; SpO2, Peripheral capillary oxygen saturation.

$\mathrm{b}$ Data are presented as mean $\pm \mathrm{SD}$.

Figure 1. Comparison of Patient's Response to Sedation by Midazolam (MIDA) and Dexmedetomidine (DEX)

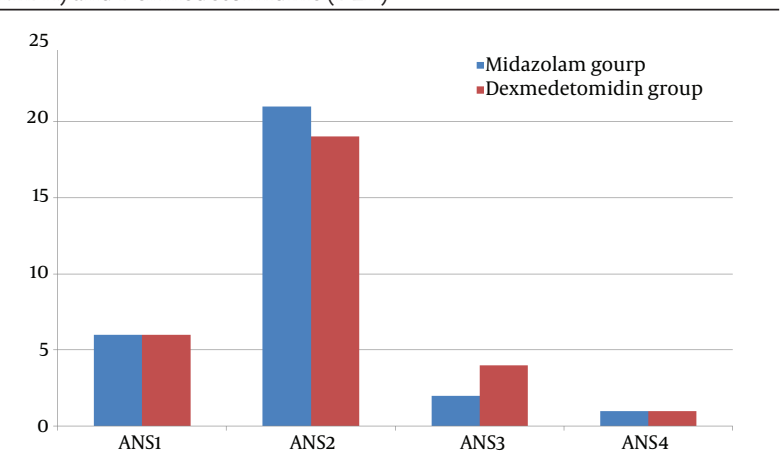

$\mathrm{P}<0.05$ vs. DEX; ANS1, anesthesia score 1; ANS2, anesthesia score 2; ANS3, anesthesia score 3; ANS4, anesthesia score 4.

Figure 2. Anesthesia Score of Two Groups, Midazolam (MIDA) and Dexmedetomidine (DEX) are Compared Together

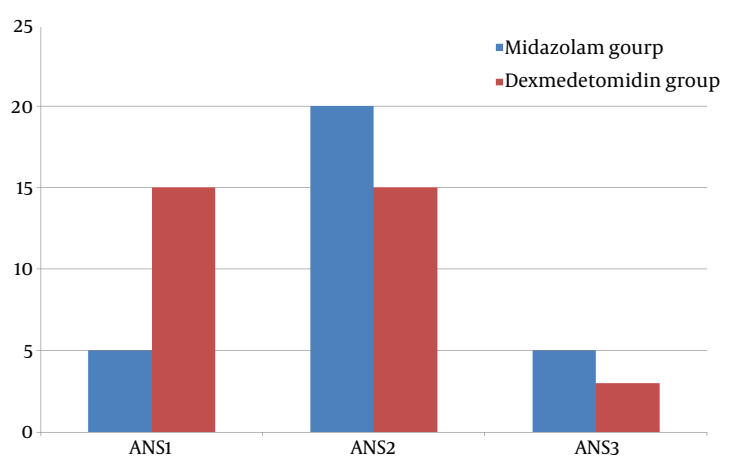

$\mathrm{P}<0.05$ vs. MIDA; ANS1, Calm and cooperating; ANS2, Anxious but without resistance; ANS3, Anxious with slight resistance.

\section{Discussion}

In the present study, we compared the effects of dexmedetomidine, an agent being widely used for analgesia in children, with midazolam, used widely as premedication in children's anesthesia practice, with respect to their safety and efficacy. Patients received $0.2 \mu \mathrm{g} / \mathrm{kg}$ of dexmedetomidine or $0.5 \mathrm{mg} / \mathrm{kg}$ of midazolam orally and screened for any side effects, preoperational anxiety, and mask acceptance behavior. Results revealed that both agents similarly ease anxiety and preoperational distress during separation from parents. Dexmedetomidine improved mask acceptance behavior compared to midazolam. Both drugs had no remarkable side effects. However, one case of hiccups was seen during midazolam administration.

Children anxiety during separation from parents before anesthesia is a major issue in children anesthesia practice, especially in cardiac surgery $(6,11)$. It is now well established that premedication to relieve anxiety during separation from parents and improve the mask acceptance behavior is an important measure to achieve better anesthesia and reduce the stress of the patient (3). However, it is a matter of debate which approach and pharmacological agents are appropriate for this purpose. Several agents such as midazolam, clonidine, and dexmedetomidine have been studied for their desirable properties in reducing stress and psychological trauma in pediatrics surgery $(4,7,12,13)$.

Sheta et al. (7) compared the intranasal midazolam vs. dexmedetomidine as premedication in children anesthesia. They reported that intranasal dexmedetomidine $1 \mu \mathrm{g} / \mathrm{kg}$ has better sedative effects compared to 0.2 
$\mathrm{mg} / \mathrm{kg}$ intranasal midazolam. A study by Talon et al. (5) revealed that intranasal dexmedetomidine (2 microg/kg) premedication has similar effects with oral midazolam $(0.5 \mathrm{mg} / \mathrm{kg})$ on preoperative anxiety. Another study by Yuen et al. (8) revealed that 0.5 or $1 \mu \mathrm{g} / \mathrm{kg}$ doses of dexmedetomidine and $0.5 \mathrm{mg} / \mathrm{kg}$ of midazolam are similarly as premedication. According to their results, dexmedetomidine induced more sedation, however, its efficacy to improve cooperation of children was similar to midazolam. Our findings were very similar to the findings of these studies and showed that dexmedetomidine induces good sedation, is safe and without remarkable adverse effects. It successfully eases the anxiety on separation from parents. This effect is comparable with midazolam, although its effect on mask acceptance behavior was superior to midazolam. This is in compliance with previous studies. As cost of treatment with dexmedetomidine is higher than midazolam, it is suggested that the treatment to be customized to each patient's history and condition. Particularly, considering some known serious effects of midazolam to block explicit memory and preserving implicit memory.

A recent study by Verma et al. (14) compared the midazolam nasal spray with its oral administration as premedication in children. Their data provided evidence was indicative of better acceptance of oral administration. Therefore, in the present study, we used the oral administration route as the children are less likely to resist receiving the premedication itself.

In sum, according to the results of the present study and considering the evidence from other studies discussed here, it appears that oral premedication with dexmedetomidine, 45 minutes before transferring the patient to the operating room is superior to midazolam as premedication in children anesthesia in cardiac surgery, however, the benefits of using this agent must outweigh its higher cost.

\section{Acknowledgements}

We cordially appreciate the staff of Rajaei Heart Center (Tehran-Iran) and our patients and their family that without their support, this research was not possible. We also extend our warmest regards to the Rajaei Heart Center for financially supporting this research.

\section{Authors' Contributions}

Conduct of study: Seyedeh Zahra Faritus, Mohsen Ziyaeifard: administration technical and scientific revision of the article; Mohammad javad Mehrabanian: literature search and clinical analysis; Mehrdad Khazaei-Koohpar data interpretation and critical revision of the article data collection and manuscript preparation.

\section{References}

1. Mensah GA, Forouzanfar MH, Naghavi M, Lozano R, Ezzati M Moran A, et al. Comparable estimates of mortality and trends for cardiovascular diseases including congenital heart disease in 21 world regions in 1990 and 2010: The global burden of diseases, injuries and risk factors study. Am J Cardiol. 2013;61(10).

2. van der Linde D, Konings EE, Slager MA, Witsenburg M, Helbing WA, Takkenberg JJ, et al. Birth prevalence of congenital heart disease worldwide: a systematic review and meta-analysis. J Am Coll Cardiol. 2011;58(21):2241-7.

3. Rosenbaum A, Kain ZN, Larsson P, Lonnqvist PA, Wolf AR. The place of premedication in pediatric practice. Paediatr Anaesth. 2009;19(9):817-28.

4. Savla JR, Ghai B, Bansal D, Wig J. Effect of intranasal dexmedetomidine or oral midazolam premedication on sevoflurane EC50 for successful laryngeal mask airway placement in children: a randomized, double-blind, placebo-controlled trial. Paediatr Anaesth. 2014;24(4):433-9.

5. Talon MD, Woodson LC, Sherwood ER, Aarsland A, McRae L, Benham T. Intranasal dexmedetomidine premedication is comparable with midazolam in burn children undergoing reconstructive surgery. J Burn Care Res. 2009;30(4):599-605.

6. Ghali AM, Mahfouz AK, Al-Bahrani M. Preanesthetic medication in children: A comparison of intranasal dexmedetomidine versus oral midazolam. Saudi J Anaesth. 2011;5(4):387-91.

7. Sheta SA, Al-Sarheed MA, Abdelhalim AA. Intranasal dexmedetomidine vs midazolam for premedication in children undergoing complete dental rehabilitation: a double-blinded randomized controlled trial. Paediatr Anaesth. 2014;24(2):181-9.

8. Yuen VM, Hui TW, Irwin MG, Yuen MK. A comparison of intranasal dexmedetomidine and oral midazolam for premedication in pediatric anesthesia: a double-blinded randomized controlled trial. Anesth Analg. 2008;106(6):1715-21.

9. Sun Y, Lu Y, Huang Y, Jiang H. Is dexmedetomidine superior to midazolam as a premedication in children? A meta-analysis of randomized controlled trials. Paediatr Anaesth. 2014;24(8):863-74.

10. Ramsay MA, Savege TM, Simpson BR, Goodwin R. Controlled sedation with alphaxalone-alphadolone. BrMed J.1974;2(5920):656-9.

11. Hamid M, Khan MA, Khatri A, Akhtar I. Effectiveness of premedication at the time of separation from parent and mask induction in paediatric patients coming for congenital heart disease surgery. J Coll Physicians Surg Pak. 2012;22(5):280-4.

12. Khatavkar SS, Bakhshi RG. Comparison of nasal Midazolam with Ketamine versus nasal Midazolam as a premedication in children. Saudi J Anaesth. 2014;8(1):17-21.

13. Mitra S, Kazal S, Anand LK. Intranasal clonidine vs. midazolam as premedication in children: a randomized controlled trial. Indian Pediatr. 2014;51(2):113-8.

14. Verma RK, Paswan A, De A, Gupta S. Premedication with midazolam nasal spray: an alternative to oral midazolam in children. Anesth Pain Med. 2012;1(4):248-51. 